\title{
Ernährungstherapie in China und Perspektiven im Westen
}

\author{
Ute Engelhardt \\ Department für Asienstudien/Institut für Sinologie der Ludwig-Maximilians-Universität München, Deutschland
}

$D^{2}$ as Wissen über chinesische Medizin hat in den letzten 30 Jahren in den westlichen Ländern rapide zugenommen. Eine der letzten Therapieformen der chinesischen Medizin, die nach Akupunktur, Arzneimitteltherapie, Qigong und Tuina (chinesische manuelle Therapie) den Westen erreicht hat, ist die chinesische Diätetik.

Die Beschäftigung mit Ernährung und Medizin kann in China auf eine lange Tradition zurückblicken, und dies nicht nur im Rahmen der chinesischen Medizin, sondern auch in $\mathrm{Zu}$ sammenhang mit der „Lebenspflege“ (yangsheng 養生). Essen und Kochen waren seit jeher mehr als eine reine Notwendigkeit, sie wurden als Kunst angesehen und gehörten $\mathrm{zu}$ den wenigen Freuden des Lebens. Die grosse Bedeutung, die dem Essen beigemessen wurde, spiegelt sich unter anderem darin wider, dass von den 4000 Bediensteten, die in der Antike unter der Zhou-Dynastie (1030-221 v.u.Z.) am Königshof beschäftigt waren, 2200 für Essen und Trinken zuständig waren (Zhouli) 周禮, [1]).

\section{Enge Verbindung zwischen Ernährung und Medizin}

Von frühester Zeit an war man sich in China sehr deutlich bewusst, welche Auswirkungen die Nahrung auf den menschlichen Körper hat und wie wichtig eine ausgewogene Ernährung für das allgemeine Wohlbefinden ist. Dieser enge Zusammenhang zwischen Ernährung und Medizin lässt sich mindestens bis ins 3. Jh. v.u.Z. zurückverfolgen. In dieser frühen Zeit wurde so gut wie kein Unterschied zwischen

Während der letzten 30 Jahre hat in den westlichen Ländern das Wissen über chinesische Medizin rapide zugenommen. Eine der letzten Therapieformen, die den Westen erreicht hat, ist die chinesische Diätetik. Dieser Artikel beschäftigt sich zunächst mit der engen Verbindung zwischen Medizin und Ernährung in China sowie mit der Frage, welche unterschiedlichen Formen der Diätetik heute in China bekannt sind und wie sie definiert werden können. Darüber hinaus wird in einem kurzen historischen Abriss die Verbreitung der chinesischen Ernährungstherapie im Westen seit den 1930er Jahren dargestellt. Anschliessend werden Perspektiven der chinesischen Diätetik im Westen beleuchtet und zum Abschluss die Grundzüge der chinesischen Diätetik denen der westlichen Ernährungstherapie tabellarisch gegenübergestellt, um Vor- und Nachteile der beiden Therapieformen vergleichen zu können.

Schlüsselwörter: Ernährungstherapie, chinesische Medizin, Diätetik, Rezeption im Westen

\section{Dietetic Treatment in China and its Perspectives in the West}

During the last thirty years, knowledge of Chinese Medicine has rapidly increased in Western countries. One of its last therapy forms to reach the West was Chinese dietetics. This article investigates the close relationship between medicine and nutrition in China as well as between different forms of dietetic treatment in today's China and how these can be defined. Moreover, it gives an overview on the historic development of Chinese dietetics in the West since the 1930's, especially in Europe. Finally, this article sheds light on future perspectives of Chinese dietetics in the West and concludes with a tabular comparison of the pros and cons of Western dietary treatment and Chinese dietetics.

Keywords: Dietetic treatment, Chinese Medicine, dietetics, reception in the West

Arznei- und Nahrungsmitteln gemacht. In einem der frühesten erhaltenen Rezepturwerke der chinesischen Medizin (Wushi er bingfang 五十二病方 aus dem Mawangdui-Grab Nr. 3, 168 v.u.Z.) finden sich z. B. zahlreiche Rezepturen für Abkochungen, bei denen es sich im Grunde genommen um Kochrezepte für alltägliche Suppen handelt $[2,3,4]$. Dieser enge Zusammenhang zwischen Ernährung und Medizin spiegelt sich auch in der legendären Person des Shennong 神農 („Göttlicher Landmann") wider, der sowohl als Patron der Landwirtschaft (Darstellung eines Han-zeitlichen Grabreliefs) als auch als
Begründer der Arzneimitteltherapie gilt. Entsprechend heisst das früheste erhaltene drogenkundliche Werk, das auf das 2. Jh. n.u.Z. zu datieren ist, „Shennongs Klassiker der Drogenkunde“ (Shennong bencao jing 神農本 草經). In der damit begründeten und später sehr umfangreichen drogenkundlichen Literatur (bencao 本草) werden sowohl Arznei- als auch Lebensmittel nach den gleichen Kriterien beschrieben; diese sind Geschmacksrichtung (sapor, wei 味), Temperaturverhalten (natura, xing 性), Leitbahnbzw. Funktionskreisbezug und sonstige Wirkungen. 
Eine Unterscheidung zwischen Arzneimitteltherapie und Diätetik beginnt sich erst in der Tang-Dynastie (618907) abzuzeichnen. Von diesem Zeitpunkt an findet sich zum ersten Mal auch ein eigener Begriff für die Diätetik, die von nun an als „Ernährungstherapie“ (shiliao 食療 oder shizhi 食治) bezeichnet wird [5]. Es liegt ihr dasselbe Ordnungsschema zugrunde, auf dem auch die chinesische Diagnostik und die anderen Therapieverfahren wie Akupunktur und vor allem die Arzneimitteltherapie aufbauen.

In seinem grundlegenden Werk zur Ernährungstherapie, das in seinen „Rezepturen, die tausend Goldstücke wert sind“(Qianjin fang 千金方, Kap. 26) enthalten ist, äussert sich Sun Simiao (581-682) bereits im Jahre 650 über den Stellenwert der Diätetik innerhalb der chinesischen Medizin: „Wer nichts von der richtigen Anwendung von Lebensmitteln versteht, dem wird es nicht gelingen, sein Leben $\mathrm{zu}$ erhalten. Wer sich nicht darüber im Klaren ist, wann Arzneimittel zu meiden sind, dem wird es nicht gelingen, Krankheiten zu beseitigen. ... Denn mit Lebensmitteln kann man Qi und Xue stützen und dadurch Schrägläufigkeiten (Heteropathien, xie 邪) austreiben, die Funktionskreise festigen, die geistigen Kräfte erfreuen und den Willen stärken. Wer in der Lage ist, mit Lebensmitteln krankhafte Entgleisungen auszugleichen, Emotionen zu besänftigen und Krankheiten zu vertreiben, der darf mit Recht von sich behaupten, das ärztliche Handwerk gut zu beherrschen [6].“

\section{Unterschiedliche Formen der chinesischen Diätetik}

Im Folgenden soll der Frage nachgegangen werden, was chinesische Diätetik eigentlich ist, welche unterschiedlichen Formen der Diätetik heute in China bekannt sind und wie sie definiert werden können.

\section{Volkstümliches diätetisches Wissen}

Zunächst ist die volkstümliche Ebene zu nennen, die in allen Bevölkerungsschichten Chinas weit verbreitet ist. Sie bedient sich meistens nur
Tab. 1. Charakteristika des volkstümlichen diätetischen Wissens in China

$$
\begin{aligned}
& \text { Kennzeichen } \\
& \text { weit verbreitet } \\
& \text { wenig differenzierte Diagnostik } \\
& \text { basiert auf vor allem auf mündlicher } \\
& \text { Überlieferung } \\
& \text { ainfache Zubereitungsformen } \\
& \text { ähnlichkeit / Vergleich mit Hausmitteln }
\end{aligned}
$$

wenig differenzierter diagnostischer Methoden und unterscheidet lediglich zwischen „Kälte“ (algor, han 寒) und „Hitze“ (calor, re 熱) sowie energetischer Schwäche (depletio, xu 虚) und energetischer Überladung (repletio, shi 實). Das volkstümliche diätetische Wissen basiert bei den Lebensmittelbeschreibungen vor allem auf mündlicher Überlieferung. Das heisst, bezüglich der Wirkbeschreibungen der einzelnen Lebensmittel (Temperaturverhalten (natura, xing) und Geschmacksrichtungen (sapor, wei)) gibt es keine konkreten Festlegungen, vielmehr können sie z.B. regional stark variieren. Ein weiteres Kennzeichen dieser Art der Diätetik sind einfache Zubereitungsformen, darunter viele Gesundheitssuppen. Am ehesten lässt sich diese Ebene des diätetischen Wissens mit unseren altbewährten Hausmitteln vergleichen. Allerdings ist sie in China deutlich weiter verbreitet und blickt auf eine relativ ungebrochene Tradition zurück. In Tabelle 1 sind die Charakteristika des volkstümlichen diätetischen Wissens in China zusammengefasst.

\section{Die „kulinarische Diätküche“ Yaoshan 藥膳 und ihre Restaurants} Als zweite Ebene ist die seit über 20 Jahren sehr populäre und kommerziell erfolgreiche Ebene der diätetischen Restaurants (Yaoshan-Restaurants) zu unterscheiden.

\section{Zum Begriff Yaoshan}

Der Begriff Yaoshan 藥膳 setzt sich aus den beiden chin. Zeichen yao 藥 und shan 膳 zusammen.

Bis heute wird das Zeichen yao 藥 verwendet, um Arzneimittel, Agenzien oder Drogen mit starker arzneilicher Wirksamkeit manchmal sogar Giftigkeit zu bezeichnen.
Die grafische Darstellung des chinesischen Zeichens für shan 膳 (Delikatessen, besondere Gerichte) kann in zwei Teile geteilt werden: links "Fleisch" und rechts "excellent, ausgezeichnet”. In den "Riten der Zhou" (Zhouli 周 禮) wird shan als „Gerichte“ (vor allem Fleisch und Gemüse) bezeichnet, die zur Abrundung des Speisezettels dienen und stets von fan 飯, gekochtem Getreide (wie gekochter Reis) begleitet werden, das die Grundlage der Ernährung darstellt. Deshalb entspricht shan im modernen Sprachgebrauch dem heute üblichen cai 菜 für „Gerichte“ allgemein [3,12]. Später wurde der Begriff shan 膳 vor allem für besonders verfeinerte Gerichte verwendet, die nur der Oberschicht zugänglich waren [7].

Aus der Analyse wird ersichtlich, dass der Begriff Yaoshan eine Kombination von Arzneimitteln mit verfeinerten, luxuriösen Lebensmitteln (wie besondere Fleischarten) und ausgewählten Zubereitungsmethoden beinhaltet. Aus diesem Grund habe ich als Übersetzung „kulinarische Diätküche“ gewählt.

Heute wird Yaoshan 藥膳 gewöhnlich wie folgt definiert: „Arzneimittel und Lebensmittel werden nach der Theorie der Traditionellen Chinesischen Medizin miteinander kombiniert und sowohl mit traditionellen Kochmethoden als auch mit speziellen Aufbereitungsmethoden behandelt, um schmackhafte und optisch ansprechende Gerichte herzustellen, die zugleich die Gesundheit erhalten und therapeutisch wirksam sind" [13].

\section{Entwicklung der Yaoshan-Restaurants} $\mathrm{Zu}$ Beginn der 1980er-Jahre zeichnete sich in der chinesischen Bevölkerung ein zunehmendes Interesse an gutem und gesundem Essen ab, und dies sowohl in ländlichen als auch in städtischen Gebieten. Nach der ökonomischen Stagnation und dem sozialen und politischen Chaos der Kulturrevolution (1966-76) hatten unter der Regierung von Deng Xiaoping weitreichende ökonomische Reformen begonnen. War die jahrhundertealte, traditionelle chinesische Ernährungsweise vor allem durch den Verzehr von Getreide und Gemüse geprägt [7], war nun ein dramatischer Anstieg im Verzehr von Fleisch, Milch- 


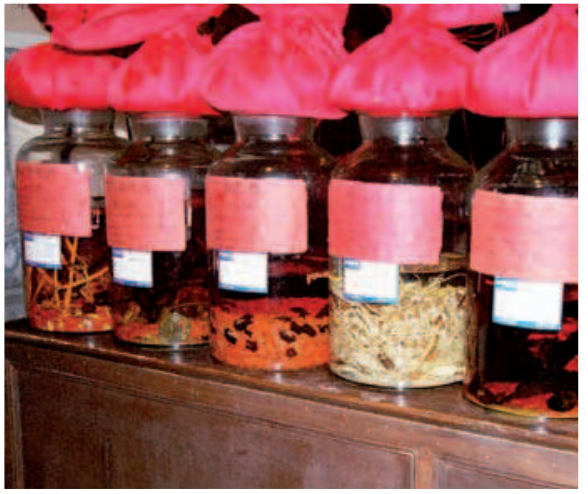

Abb. 1. Yaoshan-Weine

produkten, Geflügel, Fisch und anderen Lebensmitteln zu verzeichnen, die früher, vor allem auf dem Land, als absoluter Luxus galten [8]. Ausserdem wurde das Spektrum der erhältlichen Lebensmittel erheblich erweitert, was zu der Frage führte, welche Lebensmittel der Gesundheit besonders zuträglich sind.

Dies führte zur Gründung der „,kulinarischen Diätküche" und ihren Yaoshan-Restaurants, deren Entwicklung in den frühen 1980er-Jahren mit wenigen Restaurants begann [9]. Sie erreichte ihren Höhepunkt in den Jahren 1990 bis 1993. Parallel zu der rasanten ökonomischen Entwicklung, der Öffnung freier Märkte und der grundlegenden Verbesserung der Ernährungssituation in China [8] erhöhte sich die Anzahl der Yaoshan-Restaurants auf 900.

Auch heute noch gibt es in ganz China, vor allem jedoch in grösseren Städten, Yaoshan-Restaurants. Inzwischen bieten jedoch auch zahlreiche „normale" Restaurants, Bars und Teehäuser einige Yaoshan-Gerichte oder die äusserst populären Yaoshan-Weine an (Abb. 1). Es haben also viele YaoshanRezepte Eingang in die alltäglichen Essensgewohnheiten gefunden.

Im Zuge dieser spektakulären Entwicklung der Yaoshan-Restaurants zu einem wichtigen ökonomischen Faktor haben sich Motivation und Zielgruppe geändert. In den 1980ern ging es in den Yaoshan-Restaurants noch darum, Teil einer allgemeinen, grundlegenden Gesundheitsversorgung $\mathrm{zu}$ sein und insbesondere den Kranken, Alten und Schwachen eine adäquate Ernährung zu ermöglichen [10]. Mit ihrer zunehmenden Verbreitung und
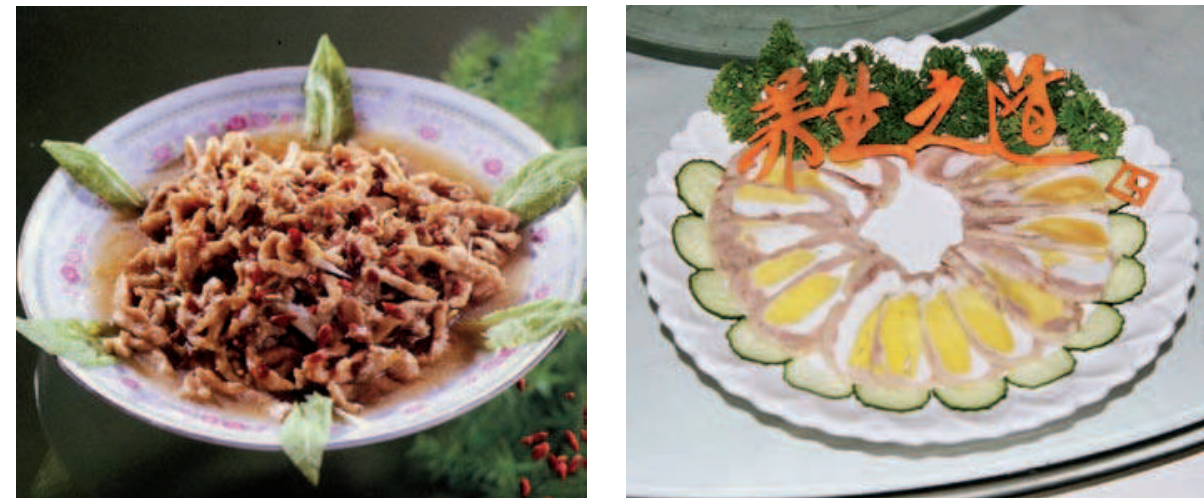

Abb. 2. Raffinierte Gerichte aus der "kulinarischen Diätküche“ (Yaoshan)

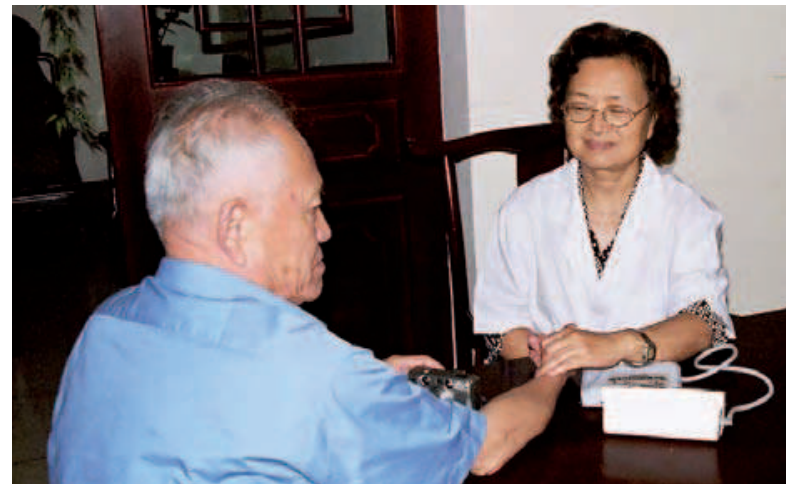

Abb. 3.

Eine Ärztin der chinesischen Medizin untersucht einen Gast in einem Yaoshan-Restaurant in Chengdu.

dem wirtschaftlichen Aufschwung Chinas wurden die Gerichte jedoch immer raffinierter, die Zutaten immer ausgesuchter und die Preise immer höher (Abb. 2).

Zugleich änderten sich die Erwartungen an die „kulinarische Diätküche“ Yaoshan: Die Gerichte sollten nicht mehr nur „stark und gross“ machen, wie in den frühen 1980ern, sondern nun wollten die Gäste durch die diätetischen Massnahmen „schlank und schlau“ werden sowie ein langes Leben erreichen. Darüber hinaus spielen in diesem Zusammenhang bei Männern die Steigerung der Potenz [11] und neuerdings bei Frauen auch kosmetische Wirkung und Schönheit sowie Gewichtsreduktion eine immer wichtigere Rolle.

Ein besonderes Kennzeichen dieser kommerziell sehr erfolgreichen „Diätküche" ist der Umstand, dass bei der Zubereitung der häufig sehr aufwändigen und raffinierten Gerichte sowohl Lebens- als auch Arzneimittel verwendet werden. Dabei bedarf es für die Zubereitung der meisten Arzneimittel spezieller Aufbereitungsmethoden, um die Schmackhaftigkeit der Speisen zu gewährleisten.

Nach dem ursprünglichen Konzept der Yaoshan-Restaurants sollte in jedem Restaurant ein TCM-Arzt sitzen (der so genannte „Arzt in der Halle“坐堂醫 生), um bei jedem Gast eine Diagnose vorzunehmen und ihn bei der Auswahl der Gerichte entsprechend zu beraten (Abb. 3). Leider gibt es keinerlei statistische Anhaltspunkte dazu, wie weit dieses Konzept tatsächlich in die Praxis umgesetzt wird. Aber unter allen Yaoshan-Restaurants, die wir bisher besucht haben, war nur eines mit einem extra dafür bestellten Arzt. In Tabelle 2 sind die Charakte-ristika von „kulinarische Diätküche“ Yaoshan 藥膳 und ihre Restaurants zusammengefasst.

\section{„Ernährungstherapie“ (shiliao 食療 oder shizhi 食治}

Als dritte Ebene ist die „Ernährungstherapie“" (shizhi oder shiliao) zu betrachten, die meistens in Kliniken oder Ambulanzen angewendet wird und auf den drogenkundlichen Beschreibungen (bencao) von Lebensmitteln basiert. Diese Form der chinesischen Diätetik lässt 
Tab. 2. Charakteristika der „kulinarischen Diätküche“ Yaoshan 藥膳 und ihrer Restaurants

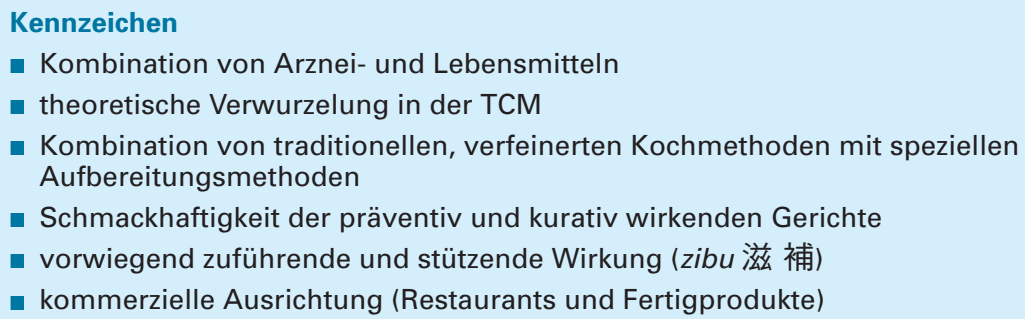

Tab. 3. Charakteristika der chinesischen „Ernährungstherapie” (shiliao 食療 oder shizhi 食治)

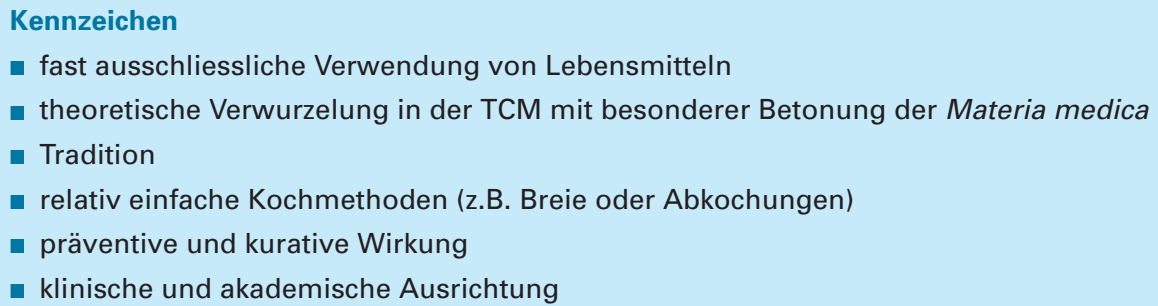

sich mindestens bis ins 6. Jh. n. Chr. zu dem bekannten Mediziner Sun Simiao (581-682) zurückverfolgen, der im 26. Kapitel seiner „Rezepte, die tausend Goldstücke Wert sind“ (Qianjin fang) den Begriff „Ernährungstherapie“ (shizhi) erstmals verwendet hat [5].

Im Gegensatz zur rasanten Entwicklung der Yaoshan-Restaurants benötigte die „Ernährungstherapie” (shiliao) deutlich mehr Zeit, um sich in Krankenhäusern, Ambulanzen und TCMUniversitäten zu etablieren. 1980 gab es im Xiyuan-Hospital in Beijing und in der Akademie für TCM in Hangzhou erste Versuche, die „Ernährungstherapie" (shiliao) einzuführen, aber schon nach wenigen Monaten mussten diese Abteilungen wieder schliessen. Der einzige uns bekannte Ort, in dem sich die „Ernährungstherapie” (shiliao) bereits in den 1990er-Jahren erfolgreich etablieren konnte, waren die Krankenhäuser und die TCM-Universität in Chengdu in Sichuan. Inzwischen gibt es an mehreren TCM-Universitäten Ausbildungsgänge für Diätassistenten.

Die „Ernährungstherapie“ (shiliao) ist die Form der chinesischen Diätetik, die sich bisher im Westen verbreitet hat. Sie eignet sich besonders gut für die Übernahme in eine andere Kultur, da die Wirkung der als milde Therapeutika eingesetzten Lebensmittel wie die von Arzneimitteln in Hinblick auf ihre Wirkung im menschlichen Organismus beschrieben wird. Es liegt ihnen also dasselbe Ordnungsschema zugrunde, auf dem auch die chinesische Diagnostik und die anderen Therapieverfahren der chinesischen Medizin, vor allem die Arzneimitteltherapie, aufbauen. Ein weiterer Vorteil dieser Form der chinesischen Diätetik liegt darin, dass sich viele unserer westlichen Nahrungsmittel, trotz geringfügiger Unterschiede zu den chinesischen, für den Einsatz nach dem gleichen drogenkundlichen Paradigma eignen. Darüber hinaus sind die einfachen Zubereitungsformen der chinesischen „Ernährungstherapie“ (shiliao) (wie Getreidebreie, Reis- oder Gemüsepfannen) auch ohne Weiteres in unserer Kultur und Küche umzusetzen. Deshalb muss niemand von heute auf morgen rein „chinesisch“ kochen. In Tabelle 3 sind die charakteristischen Kennzeichen der „Ernährungstherapie“ (shiliao) zusammengefasst.

\section{Rezeption im Westen}

Vor diesem Hintergrund soll nun in einem kurzen historischen Abriss die Rezeption der chinesischen Diätetik im Westen seit den 1930er Jahren beleuchtet werden. Sie erfolgte zunächst zeitgleich mit der Verbreitung der Arzneimitteltherapie und wurde im Wesentlichen durch den Engländer Bernard E. READ (1887-1949) angestossen, der bisher relativ wenig beachtet wurde.

Read verbrachte den grössten Teil seines Lebens in China, wo er seit seiner Ankunft im Jahre 1908 als Professor für Chemie und Pharmazie im Beijing Union Medical College (Beijing xiehe yixueyuan 北京協和醫學院) arbeitete. Er verfasste eine Reihe von Handbüchern zur Chinesischen Materia Medica und Diätetik, die lange Zeit zu den besten Publikationen auf diesem Gebiet gehörten. Dabei stützte er sich vor allem auf die „Systematische Drogenkunde“ (Bencao gangmu 本草 綱目) von Li Shizhen (1518-93). Aber in seinem Werk „Common Food Fishes of Shanghai” vermittelt er dem Leser auch ganz praktische Informationen über die gebräuchlichen Fischarten, deren Preise und ihre Nährwerte. In allen seinen Büchern gibt Read lateinische und englische Bezeichungen der Arznei- oder Lebensmittel an sowie chinesische Zeichen [14-18]. Seine Bücher zur chinesischen Materia Medica und Diätetik sind keinesfalls seine einzigen wichtigen Beiträge auf diesem Gebiet, vielmehr hat er auch an der Kompilation der ersten offiziellen Chinesischen Pharmakopoe aus dem Jahr 1930 mitgewirkt.

Ein weiterer wichtiger Schritt in der Erforschung der chinesischen Diätetik wurde von JoSEPH NEEDHAM und LU GWEI-DJEN mit ihrem Artikel „Contribution to the History of Chinese Dietetics“, in den frühen 1950er Jahren eingeleitet [19], die damit erstmals die chinesische Ernährunglehre mit ihren prophylaktischen und therapeutischen Aspekten in den Mittelpunkt des Interesses rückten.

Erst mit der zunehmenden Verbreitung der Akupunktur und der chinesischen Arzneimitteltherapie im Westen entstanden in den 1980er und 1990er Jahren einige seriöse Bücher zur chinesischen Diätetik, die vor allem zur praktischen Anwendung gedacht waren $[20,21,22]$. 
Tab. 4. Chinesische Diätetik und westliche Ernährungstherapie: Ansatz, Vorteile und Nachteile

Ansatz der westlichen Ernährungstherapie

- Gesundheit wird als Fehlen von pathologischen Störungen definiert und Diätetik als Zufuhr von Nährstoffen, die den Organismus am Leben erhalten.

- Vorwiegende Ausrichtung auf Parametern der modernen Natur- und Ernährungswissenschaft:

Nährstoffzufuhr, Blutwerte, messbare Veränderungen, Körpergewicht, Körperfettwerte

- Quantitative Ausrichtung

- Lebensmittel gelten als Nährstofflieferanten mit günstigen und ungünstigen zusätzlichen Inhaltsstoffen (Cholesterin, Transfettsäuren etc.).

\section{Vorteile der westlichen Ernährungstherapie}

- Vermeidung von Mangelernährung und Nährstoffdefiziten

- Gezielte Strategien zur Therapie von schweren Stoffwechselstörungen (z.B. Diabetes etc.)

- Genaue standardisierte Mengenangaben
Ansatz der chinesischen Diätetik

- Gesundheit wird als aktiver Prozess definiert, der zur Verfeinerung der Körperessenzen und zur Pflege der Lebenskräfte dient: Konzept der "Lebenspflege" (yangsheng)

- Vorwiegende Ausrichtung auf traditionellen Parametern wie Geschmacksrichtung (sapor, wei), Temperaturverhalten (natura, xing), Yin/Yang etc.; die subjektive Befindlichkeit des Patienten spielt eine große Rolle.

- Qualitative Ausrichtung

- Lebensmittel gelten per se als milde Therapeutika.

\section{Nachteile der westlichen Ernährungstherapie}

- Wenig Therapiemöglichkeiten bei unspezifischen Beschwerden

- Geringe Berücksichtigung von externen Einflüssen

- Starke Standardisierung, Tendenz zur Dogmatik

\section{Vorteile der chinesischen Ernährungstherapie}

- Individuell abgestimmte Auswahl der Lebensmittel unter Berücksichtigung der Vorlieben des Patienten

- Unvoreingenommener Umgang mit Fleisch, Alkohol, Kaffee, Zucker; individuelle Entscheidungen statt Dogmatik

- Berücksichtigung von Klima, Jahreszeiten, Alter

- Prophylaxe/Gesunderhaltung

- Übertragung westlicher Befunde in einen chinesischen Therapieansatz ist möglich (z.B. bei Anämie, Fettstoffwechselstörungen)

Nachteile der chinesischen Ernährungstherapie

- Kaum standardisierte Mengenangaben (Nährstoffdefizite)

- Beschränkte Wirksamkeit bei schweren Stoffwechselstörungen

- Keine Wirkungsbeschreibungen für viele westliche Lebensmittel

\section{Perspektiven der chine-} sischen Diätetik im Westen

Auch im Westen verschrieben die Ärzte des Altertums häufig Nahrungsmittel als Heilmittel, und im Zeitraum zwischen Antike und dem 16. Jh. gab es auch bei uns in der Medizin keine klare Trennlinie zwischen Lebens- und Arzneimitteln. Allerdings war die Qualifikation der Wirkung von Nahrungsmitteln nicht so kohärent in das medizinische System integriert, wie dies bei der chinesischen Medizin der Fall war. In der Neuzeit wurde dieses alte diätetische Wissen durch die moderne Ernährungswissenschaft abgelöst, die mit rein naturwissenschaftlichen Methoden die Vorgänge bei der Ernährung, der Verdauung und beim Stoffwechsel insbesondere des Menschen, aber auch der Pflanzen und Tiere untersucht. Die Ernährungsmedizin innerhalb der Westlichen Medizin ist wiederum als eine fächerübergreifende medizinische Disziplin zu sehen, die versucht, aktuelle wissenschaftliche Erkenntnisse über Physiologie und Pathophysiologie der menschlichen Ernährung zur Prävention, Heilung und Linderung von Krankheiten zu nutzen. Dabei wird Ernährung einerseits als Versorgung des Gesunden bzw. Patienten mit Kohlenhydraten, Fetten, Eiweiss, Vitaminen und Spurenelementen, anderseits auch als Ernährungsintervention, also therapeutischer Eingriff, verstanden. Sie basiert weitgehend auf der Analyse der in den einzelnen Nahrungsmitteln enthaltenen Nährstoffe und beschränkt sich im Wesentlichen auf die Darstellung quantitativer Zusammenhänge. So empfiehlt sie beispielsweise bei Hypercholesterinämie, cholesterinhaltige Nahrung zu meiden oder sich bei Diabetes nach einer quantitativ ausgerichteten Diät zu ernähren. Erst in jüngster Zeit beginnt man, auch qualitative Wirkungen von Nahrungsmitteln zu berücksichtigen und zu untersuchen, warum z.B. Patienten auf ein und dasselbe Nahrungsmittel völlig unterschiedlich reagieren. Die moderne westliche Ernährungslehre beginnt also erst jetzt, auf regulative und funktionelle Aspekte zu achten, die in der chinesischen Diätetik seit jeher im Vordergrund stehen. Deshalb kann hier der Ansatz der chinesischen Diätetik wertvolle Anregungen bieten.

$\mathrm{Zu}$ den Perspektiven der chinesischen Diätetik im Westen lassen sich zunächst folgende Fragen stellen: Unter welchen Bedingungen ist in westlichen Ländern eine Weiterentwicklung der chinesischen Diätetik möglich? Wie lässt sich mit den kulturellen Unterschieden umgehen? In diesem Zusammenhang ist festzustellen, dass von den Patienten keineswegs gefordert werden muss, von heute auf morgen rein „chinesisch“ zu kochen, sondern dass 
vielmehr aufgrund der Einordnung der Lebensmittel nach den Kriterien der chinesischen Arzneimittelkunde auch unsere westlichen Nahrungsmittel nach dem gleichen drogenkundlichen Paradigma eingesetzt werden können. Ausserdem sind die einfachen Zubereitungsformen der chinesischen „Ernährungstherapie“ (wie Getreidebreie, Reis- oder Gemüsepfannen) auch ohne weiteres in unserer Kultur und Küche umzusetzen.

In Tabelle 4 werden die Grundzüge der chinesischen Diätetik denen der westlichen Ernährungstherapie gegenübergestellt, um Vor- und Nachteile der beiden Therapieformen vergleichen $\mathrm{zu}$ können. In diesem Zusammenhang wird der Begriff Diätetik in seiner doppelten Bedeutung verwendet: zum einen als Synonym für Ernährungslehre im Rahmen der chinesischen Medizin und zum anderen als aus dem Lateinischen (diaita, dt. Lebensweise, von gr. diaitetike) stammender Sammelbegriff, der ursprünglich alle Massnahmen umfasste, die zur Gesunderhaltung oder Heilung beitragen, sowohl körperlich als auch seelisch, im Sinne einer geregelten Lebensführung.

Vor diesem Hintergrund erscheint es als erstrebenswert, beide Konzepte sinnvoll miteinander zu verbinden, was vielerorts auch bereits geschieht.
Eine sinnvolle zukünftige Entwicklung könnte in der Ausbildung einer integrativen Ernährungstherapie liegen, die durch ein öst-westliches Miteinander von traditionellem Erfahrungswissen und modernen wissenschaftlichen Erkenntnissen nicht nur die Therapeuten profitieren lässt, sondern vor allem ihre Patienten. Dazu ist es notwendig, einerseits die Entwicklung der chinesischen Diätetik in ihrem Ursprungsland China im Blick zu behalten und zum anderen ihre Wirksamkeit anhand von klinischen Studien untersuchen.

\section{Literatur}

1. Chang K.C. ed. (1977). Food in Chinese Culture, Yale University Press, New Haven and London 1977.

2. Harper, Donald (1998). Early Chinese Medical Manuscripts: The Mawangdui Medical Manuscripts. Wellcome Asian Medical Monographs, London.

3. Huang, H.T. (1990). "Han Gastronomy - Chinese Cuisine in statu nascendi" in Interdisciplinary Science Reviews, Vol.15, No.2:139152.

4. Huang, H.T. (2000). Fermentation and Food Science. Science and Civilization in China, Volume 6, Part 5, Cambridge University Press, 2000.

5. Engelhardt, Ute. (2001). "Dietetics in Tang China and the first extant works of materia dietetica" in ed. by. E. Hsu, Cambridge University Press, Needham Research Institute Studies 3, Cambridge 2001, S. 173-192.

6. Engelhardt, Ute und Nögel, Rainer (2008). Rezepte der chinesischen Diätetik, Elsevier, Urban \&Fischer, München, Wien, Baltimore 2008 .

\section{Weiterführende Informationen und Ausbildungsadressen}

\section{Internationale Gesellschaft für Chinesische Medizin / SMS Societas Medicinae Sinensis} Die SMS ist eine der ältesteten deutschsprachigen Gesellschaften für chinesische Medizin. Sie bildet seit 30 Jahren in allen Aspekten der chinesischen Medizin aus, auch in der Schweiz unter der ehemaligen SAGA und heutigen SACAM (www.sacam.ch).

Aus- und Fortbildungen in Diätetik (auch Spezialkurse und diätetisches Kochen) sowie in Qigong, Taiji und Tuina

Tel. +49 (0)89759057 85, Fax: +49(0)8975905786

Ärztliche Ausbildung in Chinesischer Medizin (mit Kursen in Akupunktur, Diagnostik, Phytotherapie und klinischen Kursen, umfangreiches Gesamtangebot von über 1000 Stunden)

Franz-Joseph-Str. 38, DE-80801 München

Tel: +49 (0)89388 880 31, Fax: +49 (0)89388 88066

sms@tcm.edu n www.tcm.edu

\section{Private Universität Witten/Herdecke GmbH}

Fachbereich Chinesische Medizin, Frau Wessel

Alfred-Herrhausen-Straße 50, DE-58448 Witten

Tel. +49 (0)2302 926 705, Fax +49(0)2302926707

tcm@uni-wh.de $\square$ www.dwgtcm.de

\section{DÄGfA - Deutsche Ärztegesellschaft für Akupunktur e.V.}

Auch die DÄGfA bietet einzelne Kurse für Chinesische Ernährung an.

Würmtalstraße 54, DE-81375 München

Tel: +49 (0)89 71005 11, Fax: +49 (0)89 7100525

fz@daegfa.de . www.daegfa.de
7. Sabban, Francoise (1993). „La viande en Chine: imaginaire et les usages culinaires", in Anthropozoologica 1993/18, p.79-90.

8. Smith, Christopher (1993). „(Over)eating success: the health consequences of the restoration of capitalism in rural China", in Social Science and Medicine Vol.37, No.6, S.761-770.

9. Engelhardt, Ute. (1998). "The Development of the Yaoshan 藥膡 (Refined Medical Cuisine) and its Restaurants", in Zhang Yuxin ed. Diwuci zhongguo yinshi wenhuaxueshu yantaohui lunwenji 第五次中國飲食文化學術研 討會論文集 ( Symposiums über die diätetische Kultur Chinas"), Foundation of Dietary Culture Taibei 1998, S. 71-89.

10. Peng Mingquan 烹銘泉 (1994). Zhongguo yaoshan daquan 中國藥瞨大全, expanded version, Chengdu 1994.

11. Farquhar, Judith (1994). „Eating Chinese Medicine", in Cultural Anthropology 9(4) 1994, pp. 471-497.

12. Zhou Guangwu 周光武 (1984). Zhongguo pengrenshi jianbian中國烹飪史簡便，Guangzhou 1984.

13. Wang Zheyue 王者悦 ed. (1992). Zhongguo yaoshan dacidian 中國藥膪大辭典, Beijing 1992.

14. Read, Bernard E. u.a. (1948). Shanghai Foods, Shanghai 1948.

15. Read, Bernard E. (1977a). Chinese Materia Medica: a. Turtle and Shellfish Drugs, b. Avian Drugs, c. A Compendium of Minerals and Stones, Shanghai 1937, 1932, 1936, reprint in 1 Vol., Taipei 1977

16. Read, Bernard E. (1977b). Chinese Medical Plants from the Pen Ts'ao Kang Mu, Shanghai 1936, reprint, Taipei 1977.

17. Read, Bernard E. (1977c). Famine Foods listed in the Chiu Huang Pen Tsiao, Ephedra, Common Food Fishes of Shanghai, Shanghai 1946. Peking 1930, Shanghai 1939, reprint in 1 Vol., Taipei 1977

18. Sivin (1982) Preface to Chinese Medicine Series, Southern Materials Center, Taipei.

19. Lu Gwei-djen und Needham, J. (1977). "A Contribution to the History of Chinese Dietetics", Isis 1951/42, S. 13-20.

20. Eyssalet, J.M. et al. (1984). Diététique Énergétique et Médecine chinoise, 2 vol., Henri Viaud, Sisteron 1984

21. Liu Jilin, Peck, G. (ed.) (1995). Chinese Dietary Therapy, Churchill Livingstone, New York 1995.

22. Engelhardt, Ute and Hempen, C.H. (1997). Chinesische Diätetik, Urban \& Schwarzenberg, München, Wien, Baltimore 1997, third edition 2006

\section{Disclosure Statement}

The author declares that no financial or other conflict of interest exists in relation to the content of this article.

\section{Korrespondenzadresse:}

Dr. phil. Ute Engelhardt

Vizepräsidentin der Internationalen Gesellschaft für Chinesische Medizin (SMS)

Fakultät für Kulturwissenschaften Department für Asienstudien Institut für Sinologie Ludwig-Maximilians-Universität Kaulbachstrase 51a, DE-80593 München u.engelhardt@tcm.edu 